\title{
Spinocerebellar ataxia type 6 (SCA6): Clinical pilot trial with gabapentin
}

\author{
Katsuya Nakamura ${ }^{1}$, Kunihiro Yoshida ${ }^{1}$, Daigo Miyazaki ${ }^{1}$, \\ Hiroshi Morita $^{1}$, Shu-ichi Ikeda ${ }^{1}$
}

\footnotetext{
${ }^{1}$ Department of Medicine (Neurology and Rheumatology), Shinshu University School of Medicine
}

Corresponding author: Kunihiro Yoshida, M.D.

Department of Medicine (Neurology and Rheumatology), Shinshu University School of Medicine, 3-1-1 Asahi, Matsumoto 390-8621, Japan

TEL: $+81-263-37-2673$

FAX: $+81-263-37-3427$

e-mail: kyoshida@shinshu-u.ac.jp 


\begin{abstract}
The clinical effect of the GABAergic drug gabapentin was evaluated in 11 patients with spinocerebellar ataxia type 6 (SCA6). The total period of gabapentin treatment was 4 weeks, and outcome measures were determined with the International Cooperative Ataxia Rating Scales (ICARS) and postural sway studies. At week 4, 5 patients showed a decrease of the ICARS values by more than $10 \%$ compared with the pre-treatment baseline. Eight patients showed a more than 10\% decrease of the sway area (SA) and/or sway path length (SPL) values in postural sway studies. The ICARS values and SA/SPL values were not necessarily consistent in each patient, but 3 patients showed a more than $10 \%$ decrease in the ICRAS, SA, and SPL values at week 4 when compared to the pre-treatment baseline. As a whole, the efficacy of gabapentin was not statistically confirmed in the 4-week trial because of the variation in efficacy in each patient, but the data are indicative that some SCA6 patients could benefit from gabapentin treatment.
\end{abstract}

Key words: spinocerebellar ataxia type 6 (SCA6), gabapentin, the International Cooperative Ataxia Rating Scales (ICARS), voltage-dependent calcium channel (VDCC), $\gamma$-aminobutyric acid (GABA), postural sway study 


\section{Introduction}

Spinocerebellar ataxia type 6 (SCA6) is a neurodegenerative disorder of autosomal-dominant inheritance characterized by a slowly progressive ataxia and dysarthria $[1,2]$. It is caused by an abnormal expansion of a trinucleotide (CAG) repeat in exon 47 of the human $\alpha 1 \mathrm{~A}$ voltage-dependent calcium channel subunit gene (CACNA1A) on chromosome 19p13 [3, 4]. SCA6 is one of the most prevalent subtypes of autosomal dominant cerebellar ataxia (ADCA) and accounts for 10-33\% of ADCA in Japan $[1,2,5]$.

Voltage-dependent (or voltage-gated) calcium channels (VDCCs or VGCCs) are hetero-oligomeric proteins consisting of the pore-forming $\alpha_{1}$ and auxiliary $\beta, \alpha_{2} \delta$, and in some tissues $\gamma$ subunits [6-9]. VDCCs mediate calcium influx into cells in response to changes in membrane potential. Disturbances of VDCCs cause a number of neurological diseases, such as epilepsy, migraine, and cerebellar ataxia [6-9]. An expanded CAG repeat in CACNA1A results in a lengthened polyglutamine (polyQ) tract in the carboxyl terminal of the $\alpha 1 \mathrm{~A}$ subunit $[3,4,6,9]$. It is postulated that heterologous expression of mutated $\alpha 1 \mathrm{~A}$ subunits enhances calcium channel dysregulation, and interferes with calcium homeostasis in Purkinje cells [6, 9-11].

Several drugs, stereotactic neurosurgery, and transcranial magnetic stimulation have been used in an attempt to relieve the symptoms of SCA, including SCA6 [12]. Some of them have been shown to be effective in reducing the symptoms associated with SCA. Stereotactic neurosurgery has been shown to be an especially useful therapeutic option for disabling tremor in SCA patients $[13,14]$. Among the drugs for SCA, protirelin tartrate or taltirelin hydrate is the most widely used for cerebellar ataxia. Furthermore, acetazolamide has been shown to temporarily reduce the severity of cerebellar ataxia in 
SCA6 patients [15]. But the options of drug therapy have remained very limited so far.

The drugs gabapentin and pregabalin interact with the $\alpha_{2} \delta$ subunit of the P/Q-type VDCCs (also referred to as CaV2.1), which are restricted to central and peripheral neurons and are mainly involved in the influx of calcium in Purkinje cells $[6-9,16,17]$. Gabapentin slows the rate of the inactivation of recombinant P/Q-type VDCCs, expressed in Xenopus oocytes [18]. These drugs have been reported to improve ataxia in cases of cortical cerebellar ataxia (CCA) $[19,20]$. As there is a similarity of the neuropathological findings between SCA 6 and CCA, and that gabapentin may be able to decrease the inactivation of $\mathrm{P} / \mathrm{Q}$-type VDCCs, it is postulated that gabapentin could be effective in the treatment of cerebellar ataxia in SCA6 patients $[16,17]$. This hypothesis prompted us to conduct a pilot clinical trial of gabapentin for SCA6 patients. Thus, the aim of this study was to evaluate the efficacy and safety of gabapentin in the treatment of SCA6.

\section{Patients and Methods}

\subsection{Patients}

Prior to the study, all the patients were informed of the potential benefits and risks of treatment and gave written informed consent for the study. Patient enrollment began in July 2007, and the last study visit occurred in August 2008. Eleven patients with SCA6 confirmed by genetic testing were included in this study (Table 1). Three of them did not have genetic testing, but their family members had been confirmed as having SCA6 by genetic testing. The mean age at baseline was $51.5 \pm 8.3$ years $(n=11)$ and the mean duration of illness after onset was $12.9 \pm 6.5$ years $(n=11)$, respectively (Table 1$)$. Ten of the patients had already taken taltirelin hydrate when they were enrolled in this study 
and continued it during the study.

\subsection{Study design}

This was an open-label, time-serious study. The study protocol was basically the same as previously reported by Gazulla et al [19], with slight modifications. The patients except patient 11 were admitted at least 5 days around the initiation of gabapentin administration. Briefly, single doses of 400mg of gabapentin were given orally on the first day (day 1), and then gabapentin was given three times a day, in total daily doses of $1,200 \mathrm{mg}$ from day 2 to day 28 . Therefore, the period of gabapentin treatment was exactly 4 weeks (day 1-28). The outcome of the trial was determined with the International Cooperative Ataxia Rating Scale (ICARS) [21] and postural sway studies. In addition, visual analogue scales were used to assess the patient's subjective feelings on gait unsteadiness, dysarthria, sleepiness, and dizziness on every occasion. Serum gabapentin concentration and $\gamma$-aminobutyric acid (GABA) concentration in the cerebrospinal fluid (CSF) were measured. Cerebral blood flow was evaluated with $n$-isopropyl-p- ${ }^{123}$ I iodoamphetamine $\left({ }^{123}\right.$ I-IMP) SPECT before treatment and at week 4 in 9 patients.

This research protocol was approved by the Ethical Committee of Shinshu University School of Medicine. The recommendations of the Ethics Committee were in accordance with the Declaration of Helsinki on the ethical principles for medical research involving human subjects.

\subsection{Outcome Measures}

\subsubsection{ICARS}


ICARS was performed for all the patients under baseline conditions, $3 \mathrm{~h}$ after oral administration of single doses of 400mg of gabapentin (day 1), at week 2 (around day 15), at week 4 (just before day 28), and then at week 8 ( 4 weeks after the discontinuation of gabapentin administration). The assessment was performed by the first two authors on every occasion. ICARS includes the following 4 subscores; posture and gait disturbances (maximum score, 34), kinetic functions (maximum score, 52), speech disorders (maximum score, 8), and oculomotor disorders (maximum score, 6), for a possible total of 100 points [21].

\subsubsection{Postural Sway Studies}

The gravimetric test was performed using a gravicorder (GS-3000; ANIMA Co., Shinjuku, Tokyo, Japan), a triangular foot plate with three force transducers at the corners. The patients were indicated to stand on the platform of the gravicorder in a foot-closed position and keep standing with their eyes closed. When the patients were unable to stand in that position, they were allowed to open their stance so that they could keep themselves in a standing position. Recording was started 5 to 10 seconds after the patients began standing on the plate, and data was recorded for 1 minute. The measurement was performed twice on each occasion. The parameters measured in the present study were as follows: (1) sway area $\left(\mathrm{SA}, \mathrm{cm}^{2}\right)$, expressed as the area of the region surrounded by the periphery of the center of the foot pressure trace, (2) longer total sway path length $(\mathrm{SPL}, \mathrm{cm} / \mathrm{sec})$. The postural sway test was performed under baseline conditions, at $3 \mathrm{~h}$ after the administration of gabapentin (day 1 ), at week 4 , and at week 8 , and the median values for SA and SPL were calculated on each occasion. 


\subsection{Laboratory assessments}

At week 2 (two weeks after the initiation of gabapentin administration, around day 15), peripheral blood was taken 2-3 hours after taking $400 \mathrm{mg}$ of gabapentin orally. Safety was assessed by blood for cell count, and liver and renal functions. At the same time, serum gabapentin concentration was also determined.

CSF samples were obtained by a lumbar puncture in 9 of the 11 patients. GABA concentrations in the CSF were determined before and after administration of gabapentin. All measurements were done by SRL, Inc., Tokyo.

\subsection{Statistics}

As this was a pilot study, no power analysis was performed. Changes of ICARS scores, SA, and SPL values were statistically evaluated using the paired $t$-test using the soft ware SIGMASTAT (version 2.0).

\section{Result}

\subsection{ICARS (Fig. 1)}

All the patients completed this study without obvious adverse effects, although mild sleepiness was transiently observed during the first few days of treatment in 7 patients. Five patients had favorable feelings on gabapentin treatment after the protocol was completed, but their feelings were not expressed properly on the VAS (data not shown).

The mean baseline ICARS score before treatment was $29.9 \pm 12.6$ (range of 8-48). It decreased to $27.4 \pm 10.7$ (range of 10-47) at $3 \mathrm{~h}$ after gabapentin administration (day 1) $(p=0.07$, versus pre-treatment), $26.4 \pm 11.1$ (range of 5-40) at week $2(p<0.05$, versus pre-treatment), and $26.8 \pm 11.9$ (range of 5-48) at week $4(p=0.09$, versus 
pre-treatment). At week 4, five patients showed a decrease of the total ICARS score by more than $10 \%$ of the pre-treatment baseline (Table 1). Then the mean ICARS score was increased slightly to $28.3 \pm 13.4$ (range of 5-51) at week 8 , but the difference between week 4 and week 8 was not significant $(p=0.30)$. The changes in the total ICARS scores were mainly due to the differences in the subscores in posture and gait disturbances and kinetic functions, but not in speech or oculomotor disorders.

\subsection{Postural Sway Studies (Fig. 2)}

The mean SA and SPL values appeared to parallel each other (Fig. 2). The mean baseline SA value before treatment was $14.5 \pm 9.6 \mathrm{~cm}^{2}$ (range of 5.0-35.6). It decreased to $11.6 \pm 5.5 \mathrm{~cm}^{2}$ (range of $\left.4.8-19.4\right) 3 \mathrm{~h}$ after gabapentin administration (day 1$)(p=$ 0.18 , versus pre-treatment), and $10.6 \pm 5.2 \mathrm{~cm}^{2}$ (range of 3.7-18.0) at week $4(p=0.25$, versus pre-treatment). Then the mean SA value increased to $19.9 \pm 13.0 \mathrm{~cm}^{2}$ (range of 3.4-43.8) at week $8(p<0.05$, versus week 4$)$.

The mean SPL value before treatment was $5.7 \pm 2.7 \mathrm{~cm} / \mathrm{sec}$ (range of 2.6-10.6), it then significantly decreased at day $1(4.0 \pm 1.4 \mathrm{~cm} / \mathrm{sec}$, range of $2.5-6.6, p<0.05)$, but the difference was not significant at week $4(4.3 \pm 1.9 \mathrm{~cm} / \mathrm{sec}$, range of $1.7-7.4, p=0.06$ versus pre-treatment). The value in turn increased at week $8(6.8 \pm 4.9 \mathrm{~cm} / \mathrm{sec}$, range of 1.7-18.6), when compared with the value at week $4(p=0.06)$.

At week 4, 6 patients showed a decrease of both the SA and SPL values by more than $10 \%$ of the pre-treatment baseline (Table 1). Changes in the ICARS and SA or SPL value were not necessarily correlated in each patient, but 3 patients showed more than a $10 \%$ decrease in the ICARS, SA, and SPL values at week 4 when compared to the pre-treatment baseline. 


\subsection{Laboratory evaluations and cerebral blood flow}

Routine laboratory data for peripheral blood were unchanged with gabapentin treatment. The serum gabapentin concentration at week 2 was $6.2 \pm 1.7 \mu \mathrm{g} / \mathrm{ml}(\mathrm{n}=10)$. Serum gabapentin concentration in the normal renal function group (eGFR $>90 \mathrm{ml} / \mathrm{min} / 1.73 \mathrm{~m}^{2}, \mathrm{n}=4$ ) was lower than that in the mild renal dysfunction group $\left(\right.$ eGFR $\left.60-90 \mathrm{ml} / \mathrm{min} / 1.73 \mathrm{~m}^{2}, \mathrm{n}=6\right)(4.7 \pm 0.9$ versus $7.1 \pm 1.3, p<0.05)$.

Routine laboratory data for CSF were unremarkable. The mean GABA concentration before gabapentin administration was $183.9 \pm 96.6 \mathrm{pmol} / \mathrm{ml}(67-304 \mathrm{pmol} / \mathrm{ml})$, and was significantly decreased to $110.2 \pm 35.6 \mathrm{pmol} / \mathrm{ml}(68-168 \mathrm{pmol} / \mathrm{ml})$ with gabapentin treatment $(p<0.05, \mathrm{n}=9)$ (Fig. 3). The mean GABA concentration before gabapentin treatment in these SCA6 patients was not different from that in patients with other neurological diseases $(165.1 \pm 87.2 \mathrm{pmol} / \mathrm{ml}, \mathrm{n}=17)$.

${ }^{123}$ I-IMP SPECT showed an increase of blood flow in the cerebellum in some patients, but the results were inconsistent in this study (data not shown).

\subsection{A particular case of clinical improvement (patient 6)}

Patient 6 was a 56 year-old-man, who first noticed a difficulty in running at around age 40. He developed gait ataxia and speech disturbance at around age 47. At age 50, he and his family noted head tremor or titubation. Gait ataxia and dysarthria gradually worsened, and he became unable to walk without assistance around age 53. Neurological examination at age 56 showed pure cerebellar ataxia with head and hand tremor. His speech was slurred and explosive, and often difficult to understand. He needed a walker to assist with his walking. His ICARS score was 48 before gabapentin 
treatment, and was unchanged at week 4. His SA and SPL values were $35.6 \mathrm{~cm}^{2}$ and $10.6 \mathrm{~cm} / \mathrm{sec}$ before treatment, then markedly decreased to $9.3 \mathrm{~cm}^{2}$ and $3.7 \mathrm{~cm} / \mathrm{sec}$ at week 4 , respectively. The values in turn increased to $43.8 \mathrm{~cm}^{2}$ and $8.1 \mathrm{~cm} / \mathrm{sec}$ after the discontinuation of gabapentin. He felt that his head and hand tremor were depressed and his sense of sway was reduced during gabapentin treatment.

\section{Discussion}

This study was focused on a 4-week clinical trial of the GABAergic drug, gabapentin, for SCA6 patients. SCA6 is one of the few ADCAs in which the function of the causative gene product, namely the $\alpha_{1} \mathrm{~A}$ subunit of P/Q-type VDCC, is well recognized [1-4]. In SCA6, altered function of the P/Q-type VDCC may reduce calcium influx, finally leading to Purkinje cell degeneration [6,9]. Gabapentin binds to the $\alpha_{2} \delta$ subunit of the P/Q-type VDCCs, and is supposed to modulate its calcium channel function $[8,16,17]$. Based on its pharmacological characteristics, Gazulla et al. has proposed the hypothesis that the administration of gabapentin may be of therapeutic benefit for SCA6 $[16,17]$.

In the present study, gabapentin was safely administered to the SCA6 patients, although adverse effects such as sleepiness, dizziness, headache, or diplopia were recorded when used in patients with epilepsy. We assessed the clinical effects of gabapentin using the ICARS and gravimetric tests. Both the mean values of ICARS, SA, and SPL of the gravimetric test tended to improve during the administration of gabapentin, and in turn deteriorated after the discontinuation of gabapentin. In fact, 5 of the 11 patients had a favorable feeling for gabapentin treatment after the protocol was completed. At week 4, 5 of the 11 patients showed more than a $10 \%$ decrease in the 
ICARS score when compared with the pre-treatment baseline, and 6 patients showed a decrease in both the SA and SPL values by more than $10 \%$ of the pre-treatment baseline. Three showed more than a $10 \%$ decrease of the pre-treatment baseline both in the ICARS and SA/SPL values. But the effect of gabapentin treatment was not statistically confirmed when the ICARS, SA, and SPL values were compared between the pre-treatment baseline and at week 4 .

The major reason for this is that the effect varied from patient to patient. This variation appeared not to correlate with the disease severity at the pre-treatment baseline, the duration of illness, or the patient's age. One possible factor for modifying the effect may be the serum gabapentin concentration. The mean serum gabapentin concentration in 6 patients who showed a decrease of both the SA and SPL values by more than $10 \%$ of the pre-treatment baseline at week 4 was $6.5 \pm 1.4 \mu \mathrm{g} / \mathrm{ml}$, whereas the concentration in the other patients was $5.7 \pm 2.2 \mu \mathrm{g} / \mathrm{ml}$. Serum gabapentin concentration was likely to be associated with renal function. Gazulla et al. used gabapentin in CCA patients in daily doses ranging from 900 to1,600 $\mathrm{mg}$ [19]. For the maximum clinical effects on SCA6 patients, the total daily doses of gabapentin may need to be carefully adjusted in each patient. Among the clinical signs and symptoms of SCA6, fine postural or action tremor may be more responsive to gabapentin treatment. In 5 patients who showed more than a $10 \%$ decrease in the total ICARS value at week 4 , the change mainly resulted from a decrease in the subscore for the kinetic functions. This might be caused by a reduction of postural or action tremor in the hands and legs by gabapentin treatment. Patient 6 obviously felt a reduction in his head and hand tremor during gabapentin treatment.

It is intriguing that the GABA concentration in the CSF was decreased by gabapentin 
treatment. This finding clearly indicates that gabapentin could alter GABA metabolism in the central nervous system. In addition to a modulation of VDCCs, gabapentin is postulated to activate the GABA transporter and to enhance GABA re-uptake into the nerve terminals of GABA neurons. Thus, a decrease of GABA content in CSF by gabapentin treatment may reflect an activation of GABA re-uptake into the nerve terminals. P/Q-type VDCC is considered to control neurotransmitter release, especially at excitatory synapses [9], but a modulation of GABA metabolism via an action on inhibitory synapses may be more important in terms of drug therapy for ataxic patients. The reason is that GABA is an inhibitory neurotransmitter released from Purkinje cells, which are a major efferent from the cerebellum, and inevitably affected in spinocerebellar ataxia.

The present study was not randomized or blinded, thus "the placebo effect" might modify the results. However, our data indicated that there may be SCA6 patients who respond to gabapentin treatment. With very limited options of drug therapy for SCA, gabapentin is worthy of further testing for its efficacy on SCA6 patients.

\section{Acknowledgement}

We are grateful to all the patients who participated in this study. This work was supported in part by a Grant-in-Aid for Science Research from the Ministry of Education, Science and Culture, Japan and a grant from the Research Committee for Ataxic Diseases, the Ministry of Health, Labor, and Welfare, Japan. 


\section{References}

[1] Bird TD. Hereditary ataxia overview. In: Gene Reviews: Genetic Disease Online Reviews at GeneTest-GeneClinics[database online].[cited 28 February 2008]. Copyright, University of Washington, Seattle, Available at http://www.geneclinics.org/

[2] Schöls L, Bauer P, Schmidt T, Schulte T, Riess O. Autosomal dominant cerebellar ataxias: clinical features, genetics, and pathogenesis. Lancet Neurol 2004; 3: 291-304.

[3] Zhuchenko O, Bailey J, Bonnen P, Ashizawa T, Stockton DW, Amos C, et al. Autosomal dominant cerebellar ataxia (SCA6) associated with small polyglutamine expansions in the $\alpha_{1 \mathrm{~A}}$-voltage-dependent calcium channel. Nat Genet 1997; 15: 62-9.

[4] Riess O, Schöls L, Böttger H, Nolte D, Vieira-Saecker, AMM, Schimming C, et al. SCA6 is caused by moderate CAG expansion in the $\alpha_{1 \mathrm{~A}}$-voltage-dependent calcium channel gene. Hum Molec Genet 1997; 6: 1289-93.

[5] Sasaki H, Yabe I, Tashiro K. The hereditary spinocerebellar ataxias in Japan. Cytogenet Genome Res 2003; 100: 198-205.

[6] Missiaen L, Robberrecht W, van den Bosch L, Callewaert G, Parys JB, Wuytack F, et al. Abnormal intracellular $\mathrm{Ca}^{2+}$ homeostasis and disease. Cell Calcium 2000; 28: $1-21$.

[7] Catterall WA. Structure and regulation of voltage-gated $\mathrm{Ca}^{2+}$ channels. Annu Rev Cell Dev Biol 2000; 16: 521-55.

[8] Klugbauer N, Marais E, Hofmann F. Calcium channel $\alpha_{2} \delta$ subunits: differential expression, function, and drug binding. J Bioenerg Biomembr 2003; 35: 639-47. [9] McKeown L, Robinson P, Jones OT. Molecular basis of inherited calcium 
channelopathies: role of mutations in pore-forming subunits. Acta Pharmacol Sin 2006; 27: 799-812.

[10] Matsuyama Z, Wakamori M, Mori Y, Kawakami H, Nakamura S, Imoto K. Direct alteration of the $\mathrm{P} / \mathrm{Q}$-type $\mathrm{Ca}^{2+}$ channel property by polyglutamine expansion in spinocerebellar ataxia 6. J Neurosci 1999; 19: RC14(1)-14(5).

[11] Toru S, Murakoshi T, Ishikawa K, Saegusa H, Fujigasaki H, Uchihara T, et al. Spinocerebellar ataxia type 6 mutation alters P-type calcium channel function. J Biol Chem 2000; 275: 10893-8.

[12] Fogel B, Perlman S. An approach to the patient with late-onset cerebellar ataxia. Nature Clin Pract Neurol 2006; 2: 629-35.

[13] Shimojima Y, Hashimoto T, Kaneko K, Yazaki M, Yoshida K, Goto T, et al. Thalamic stimulation for disabling tremor in a patient with spinocerebellar degeneration. Stereotact Funct Neurosurg 2005; 83: 131-4.

[14] Freund H-J, Barnikol UB, Nolte D, Treuer H, Auburger G, Tass PA, et al. Subthalamic-thalamic DBS in a case with spinocerebellar ataxia type 2 and severe tremor -a unusual clinical benefit. Mov Disord 22: 732-735, 2007.

[15] Yabe I, Sasaki H, Yamashita I, Takei A, Tashiro K. Clinical trial of acetazolamide in SCA6, with assessment using the ataxia rating scale and body stabilometry. Acta Neurol Scand 2001; 104: 44-7.

[16] Gazulla J, Tintoré M. The P/Q-type voltage-dependent calcium channel as pharmacological target in spinocerebellar ataxia type 6: gabapentin and pregabalin may be of therapeutic benefit. Medical Hypotheses 2007; 68: 131-6.

[17] Gazulla J, Tintoré M. The P/Q-type voltage-dependent calcium channel: a 
therapeutic target in spinocerebellar ataxia type 6. Acta Neurol Scand 2007; 115 : $356-63$.

[18] Kang M-G, Felix R, Campbell KP. Long-term regulation of voltage-gated $\mathrm{Ca}^{2+}$ channels by gabapentin. FEBS Lett 2002; 528: 177-82.

[19] Gazulla J, Errea JM, Benavente I, Tordesillas CJ. Treatment of ataxia in cortical cerebellar atrophy with GABAergic drug gabapentin. A preliminary study. Eur Neurol $2004 ; 52: 7-11$

[20] Gazulla J, Benavente I. Single-blind, placebo-controlled pilot study of pregabalin for ataxia in cortical cerebellar atrophy. Acta Neurol Scand 2007; 116: 235-8.

[21] Trouillas P, Takayanagi T, Hallett M, Currier RD, Subramony SH, Wessel K, et al. International Cooperative Ataxia Rating Scale for pharmacological assessment of the cerebellar syndrome. The Ataxia Neuropharmacology Committee of the World Federation of Neurology. J Neurol Sci 1997; 145: 205-11. 
Figure legends

Fig. 1 The ICARS scores

The mean ICARS scores are shown at the pre-treatment baseline, day 1, week 2, week 4 , and week 8 .

Fig. 2 Postural sway studies

The mean SA (closed column) and SPL values (open column) are shown at the pre-treatment baseline, day 1 , week 2 , week 4 , and week 8 .

Fig.3 GABA concentration in the CSF before and after gabapentin administration Data for GABA concentration before and after gabapentin administration in 9 patients are plotted. Open columns indicate the mean values for GABA concentration in the CSF before and after gabapentin administration. 
Table 1. Clinical and Demographic values of 11 SCA6 patients

\begin{tabular}{|c|c|c|c|c|c|c|c|c|c|}
\hline $\begin{array}{l}\text { Patient } \\
\text { No. }\end{array}$ & Age/Sex & $\begin{array}{l}\text { Onset of } \\
\text { disease } \\
\text { (years old) }\end{array}$ & $\begin{array}{l}\text { Duration of } \\
\text { disease } \\
\text { (years) }\end{array}$ & $\begin{array}{l}\text { CAG } \\
\text { repeat } \\
\text { number }\end{array}$ & $\begin{array}{l}\text { ICARS score } \\
\text { at baseline }\end{array}$ & $\begin{array}{l}\text { Change in } \\
\text { ICARS score } \\
(\%)^{\#}\end{array}$ & $\begin{array}{l}\text { Change in } \\
\text { SA (\%) }\end{array}$ & $\begin{array}{l}\text { Change in } \\
\text { SPL (\%) }\end{array}$ & $\begin{array}{l}\text { Serum gabapentin } \\
\text { concentration } \\
(\mu \mathrm{g} / \mathrm{ml})\end{array}$ \\
\hline 1 & $44 / \mathrm{M}$ & 34 & 10 & $\mathrm{NE}$ & 22 & 13.6 & 3.4 & -20.6 & 4.89 \\
\hline 2 & $53 / F$ & 35 & 18 & $16 / 24$ & 45 & -31.1 & 12.9 & -7.8 & 8.65 \\
\hline 3 & $39 / F$ & 29 & 10 & $12 / 23$ & 26 & -7.7 & 17 & 19.4 & 5.60 \\
\hline 4 & $41 / \mathrm{M}$ & 30 & 11 & $13 / 24$ & 37 & -2.7 & -12.8 & 1.2 & 3.50 \\
\hline 5 & $45 / \mathrm{M}$ & 34 & 11 & $\mathrm{NE}$ & 37 & -18.9 & -10.1 & -44.3 & 5.17 \\
\hline 6 & $56 / \mathrm{M}$ & 30 & 26 & $12 / 23$ & 48 & 0 & -73.9 & -66.3 & 8.32 \\
\hline 7 & $65 / F$ & 45 & 20 & $12 / 23$ & 34 & -32.4 & 128 & 30.3 & $\mathrm{NE}$ \\
\hline 8 & $57 / F$ & 44 & 13 & $14 / 24$ & 16 & -32.4 & -20.3 & -20.1 & 7.45 \\
\hline 9 & $60 / \mathrm{M}$ & 46 & 14 & $\mathrm{NE}$ & 37 & 5.4 & -78.3 & -41.9 & 6.91 \\
\hline 10 & $56 / F$ & 54 & 2 & $13 / 21$ & 8 & -37.5 & -25.5 & -33.1 & 4.68 \\
\hline 11 & $51 / \mathrm{F}$ & 44 & 7 & $12 / 23$ & 19 & 5.3 & -26.9 & -16 & 6.91 \\
\hline
\end{tabular}

\#; The value at week 4 was compared with the pre-treatment baseline 


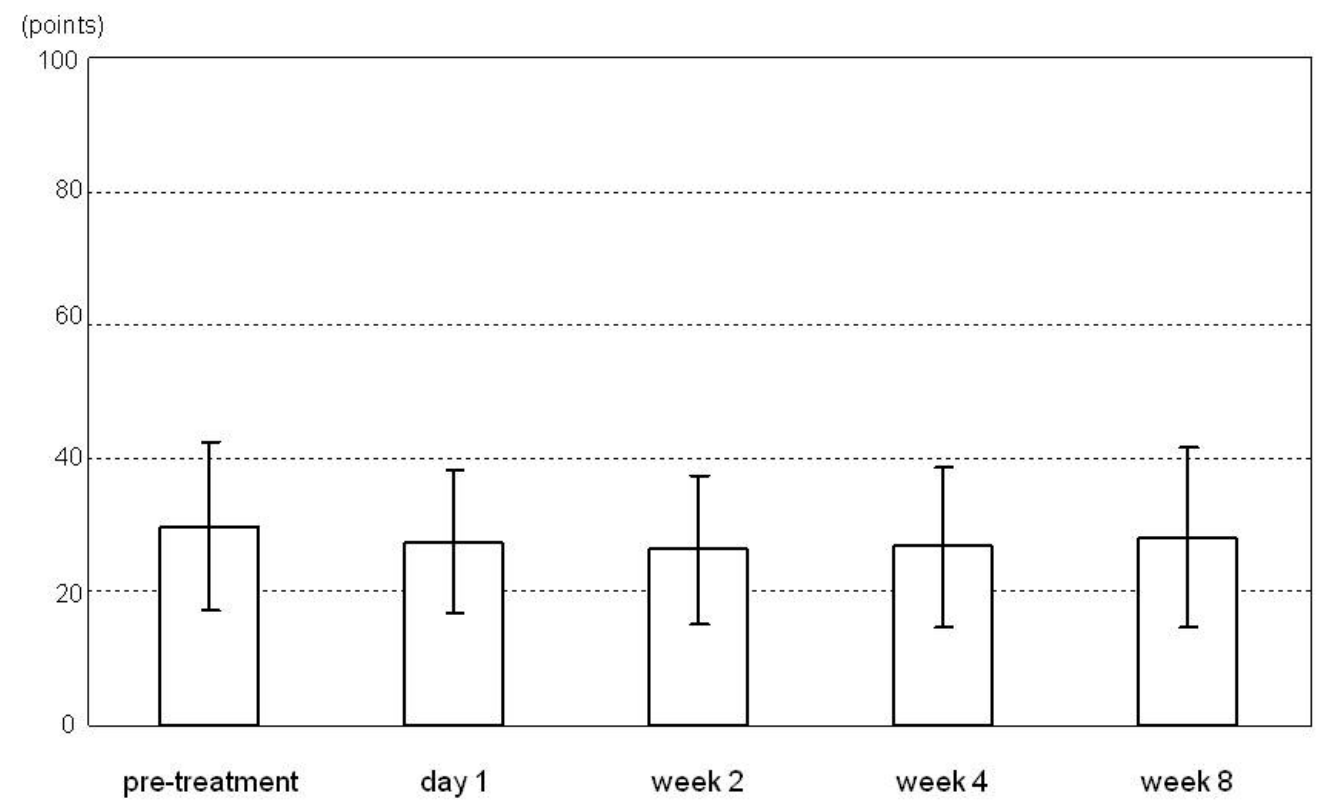

Fig. 1 


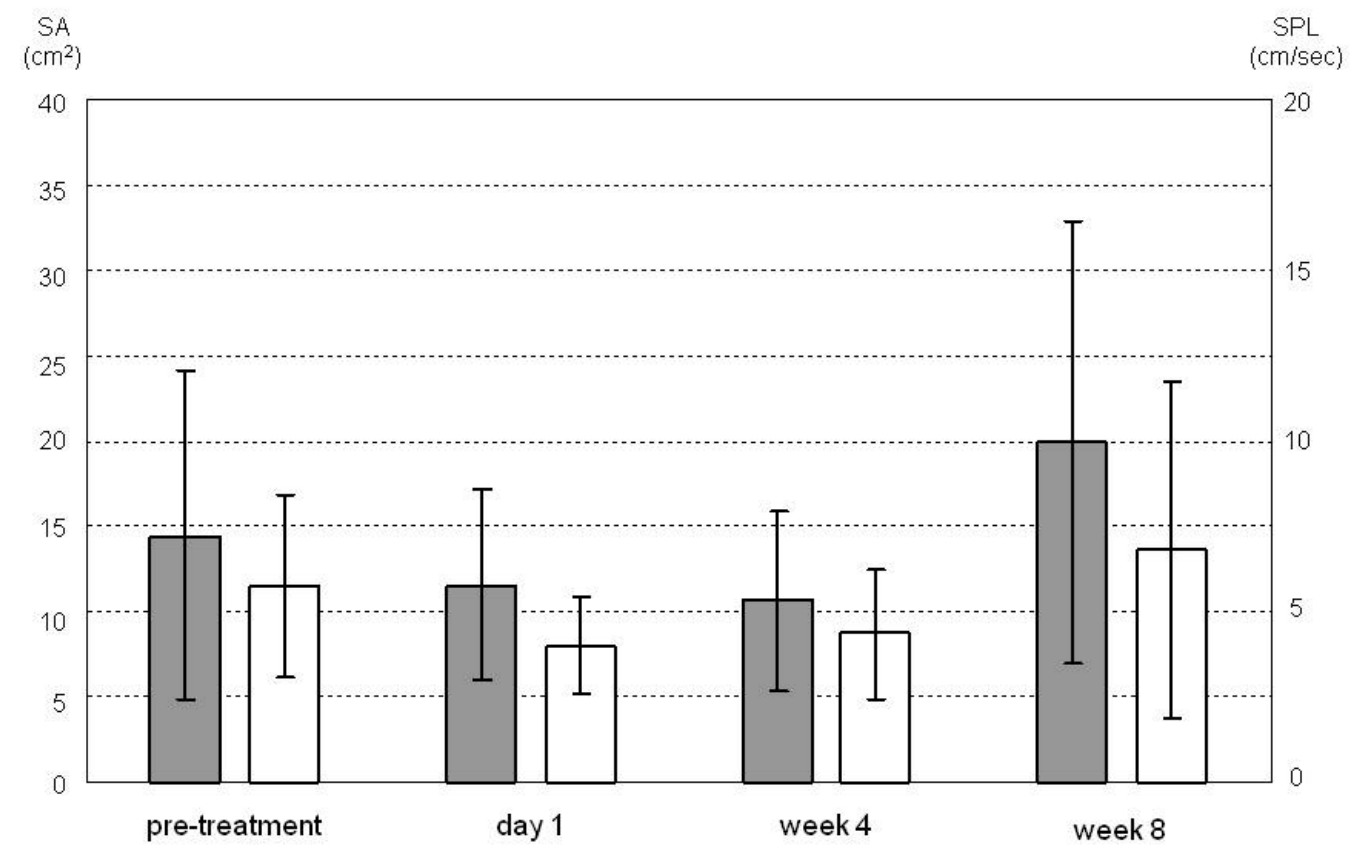

Fig. 2 


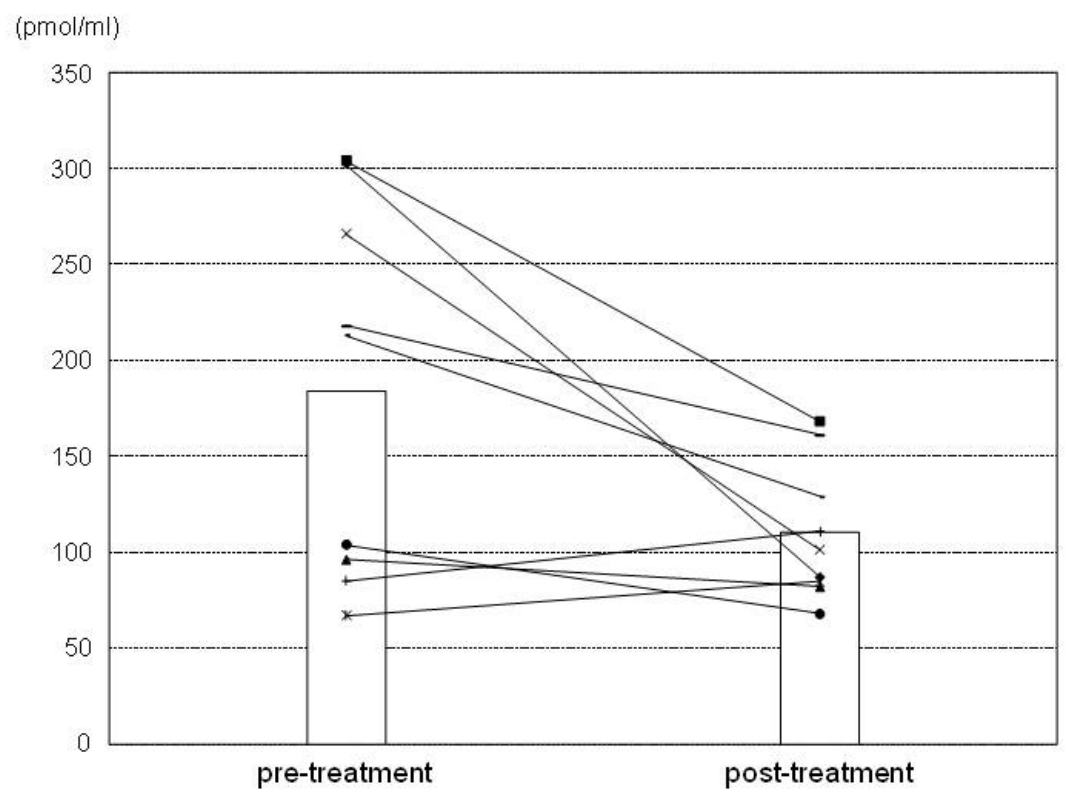

Fig. 3 